CASE RECORDS of the MASSACHUSETTS GENERAL HOSPITAL

Founded by Richard C. Cabot

Eric S. Rosenberg, M.D., Editor

Virginia M. Pierce, M.D., David M. Dudzinski, M.D., Meridale V. Baggett, M.D.,

Dennis C. Sgroi, M.D., Jo-Anne O. Shepard, M.D., Associate Editors Kathy M. Tran, M.D., Case Records Editorial Fellow

Emily K. McDonald, Sally H. Ebeling, Production Editors

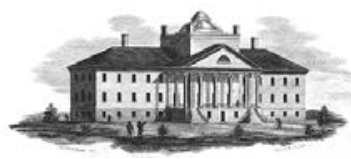

\section{Case 28-2019: A 22-Year-Old Woman with Dyspnea and Chest Pain}

\author{
Ted M. Kremer, M.D., Mark E. Lindsay, M.D., Ph.D., T. Bernard Kinane, M.D., \\ Megan H. Hawley, M.S., C.G.C., Brent P. Little, M.D., \\ and Mari Mino-Kenudson, M.D.
}

PRESENTATION OF CASE

Dr. Bethany L. Bartley (Pediatrics): A 22-year-old woman was seen in the pediatric pulmonary clinic of this hospital because of chest pain, cough, and dyspnea on exertion.

The patient had been well until she was 2 months of age, when an episode of bronchiolitis occurred. After this episode, repeated respiratory illnesses characterized by wheezing and cyanosis developed, and she was hospitalized frequently. The patient was subsequently evaluated by a pediatric pulmonologist at this hospital. Examination revealed decreased breath sounds at the apex of the right lung, and chest radiography revealed findings suggestive of congenital lobar emphysema of the right upper lobe. A blood test for alpha ${ }_{1}$-antitrypsin deficiency was negative. Transthoracic echocardiography revealed right ventricular dilatation and hypertrophy, mild pulmonary arterial hypertension, and a small patent ductus arteriosus. When the patient was 24 months of age, a right upper lobectomy, coil embolization for the patent ductus arteriosus, and a biopsy of the right middle lobe were performed.

Dr. Mari Mino-Kenudson: Histopathological examination of lung specimens revealed lobar emphysema of the right upper and middle lobes that was characterized by the presence of fewer alveoli than the number typically seen in normal lung tissue and hyperinflation of the existing alveoli (Fig. 1A). The number of bronchioles in the peripheral emphysematous areas was disproportionately low relative to the number of pulmonary vessels, and the central bronchi had mucus plugs that may have obliterated the bronchial lumens (Fig. 1B). In the hilum, the central bronchi had mucus plugs that resulted in partial obstruction of the bronchial lumens, but the amount and contour of the bronchial cartilage were normal (Fig. 1C). No diagnostic findings were consistent with pulmonary hypertension. There are two possible causes of lobar emphysema in this case: obstruction (either
From the Department of Pediatrics, UMass Memorial Medical Center, and the Department of Pediatrics, University of Massachusetts Medical School, Worcester (T.M.K.), the Departments of Pediatrics (M.E.L., T.B.K.), Radiology (B.P.L.), and Pathology (M.M.-K.), Massachusetts General Hospital, and the Departments of Pediatrics (M.E.L., T.B.K.), Radiology (B.P.L.), and Pathology (M.M.-K.), Harvard Medical School, Boston, and the Laboratory for Molecular Medicine, Partners HealthCare Personalized Medicine, Cambridge (M.H.H.) - all in Massachusetts.

N Engl J Med 2019;381:1059-67. DOI: 10.1056/NEJMcpc1904041 Copyright (C) 2019 Massachusetts Medical Society. 


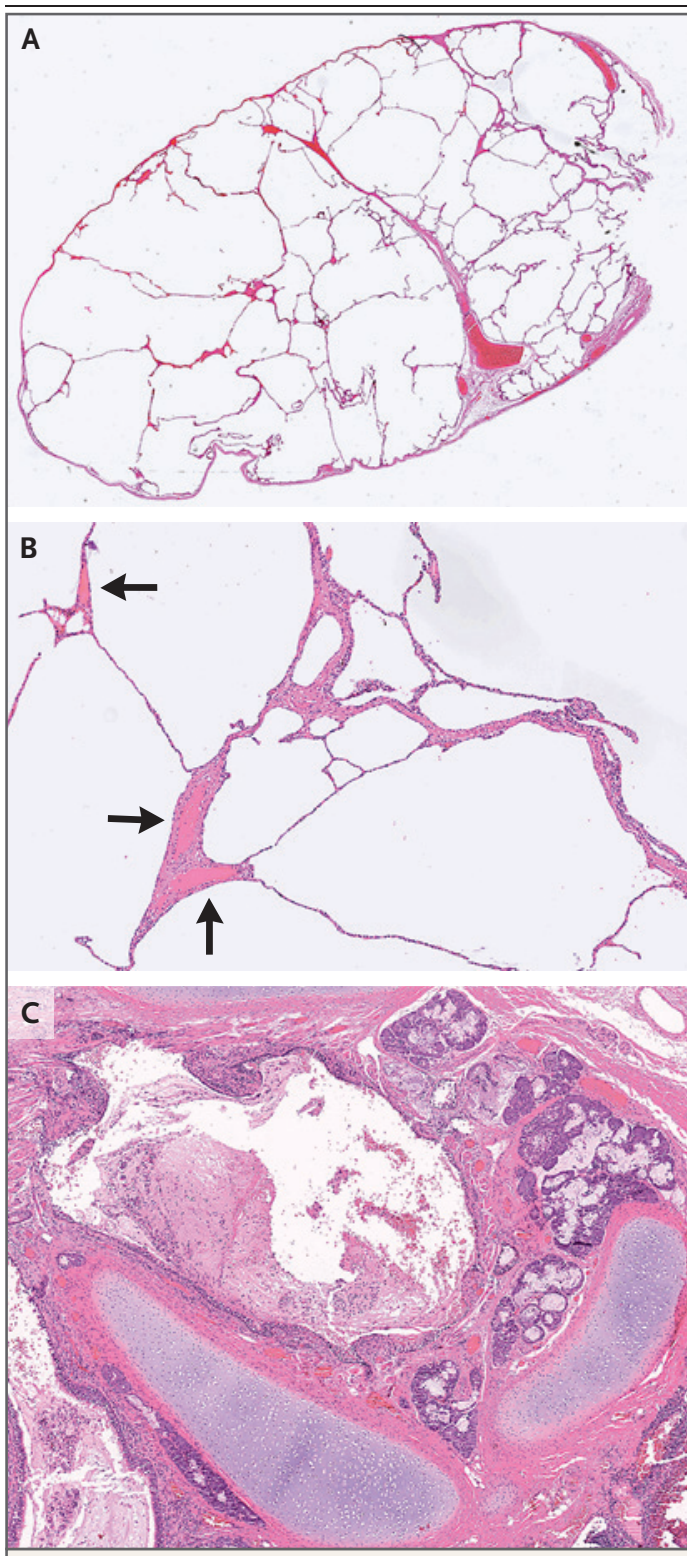

Figure 1. Lung Specimens.

On gross examination, the biopsy specimen of the middle lobe was spongelike. On microscopic examination, there was no evidence of inflammation or fibrosis, but the number of alveoli was substantially lower than the number that would typically be observed in normal lung tissue and the existing alveoli were hyperinflated. On hematoxylin and eosin staining, the simplified, uniform enlargement of air spaces is consistent with lobar emphysema (Panel A). A paucity of respiratory bronchioles in the emphysematous parenchyma is characterized by multiple isolated small pulmonary arteries (Panel B, arrows) without accompanying airways, although alveolar septa are normal. A section from the hilum shows bronchi with unremarkable immature cartilage and respiratory mucosa; mucus plugs are present, but they only partially obstruct a few central airways (Panel C). intrinsic or extrinsic) of the developing airway, and overinflation of the lung as a result of constrictive bronchiolitis that was possibly due to infection. Intrinsic obstruction is often caused by defects in the bronchial wall, such as a deficiency of bronchial cartilage or intraluminal obstruction by meconium, mucus plugs, or other substances. In this case, the bronchial cartilage was normal. Mucus plugs were observed at the time of lung resection, but they only partially obstructed the central airways. In addition, there was no evidence of constrictive bronchiolitis, although there were fewer bronchioles than normal in the area of the peripheral lung that was involved by lobar emphysema.

Dr. Bartley: The patient and her family subsequently moved from the United States to another country, and she received medical care at another hospital. When she was 6 years of age, a spontaneous pneumothorax occurred, and evaluation included genetic testing for Marfan's syndrome, which was reportedly negative. During the next 16 years, while the patient was still living in another country, dyspnea on exertion and decreased exercise tolerance slowly developed, becoming more marked in the 12 months before this presentation.

Approximately 6 weeks before this presentation, intermittent chest pain developed. The pain was sharp, could be reproduced with palpation of the affected area, and was not relieved with the administration of ibuprofen. Episodes occurred both at rest and with exertion; no precipitating or exacerbating factors were identified. The patient was seen in the emergency department of a local hospital, where an electrocardiogram was reportedly normal and a radiograph of the chest showed no pneumothorax. The patient was discharged home, and the chest pain continued to occur every few days until presentation.

On presentation to the pediatric pulmonary clinic of this hospital, the patient reported that her exercise capacity had declined during the past month and that she had become unable to climb a flight of stairs without having severe breathlessness. She also reported a 1-week history of productive cough; the sputum had initially been clear but then had become dark yellow and, on the day before this presentation, blood-tinged. The patient also had diarrhea but had no fever, chills, weight loss, vomiting, or abdominal pain. She had been born after a full-term gestation and had been delivered by cesarean section be- 
cause of breech presentation; the birth weight was $3175 \mathrm{~g}$. She had a history of depression. Medications included sertraline and inhaled albuterol as needed. She had no known allergies. The patient was fluent in English and Spanish and was an undergraduate student at a European university; she lived on campus during the academic year and spent the rest of the year at her parents' home in the United States. She did not drink alcohol, smoke tobacco, or use illicit drugs. Her younger brother had a seizure disorder and developmental delays; her mother had mild joint hypermobility, and her father was healthy.

On examination, the temperature was $37.1^{\circ} \mathrm{C}$, the pulse 82 beats per minute, the blood pressure $116 / 72 \mathrm{~mm} \mathrm{Hg}$, the respiratory rate 18 breaths per minute, and the oxygen saturation $92 \%$ while the patient was breathing ambient air at rest. The height was $169 \mathrm{~cm}$, the weight $47.7 \mathrm{~kg}$, and the body-mass index (the weight in kilograms divided by the square of the height in meters) 16.7. A blue hue was noted in the perioral region; no clubbing of the fingers and toes was seen. There was no use of accessory muscles of ventilation; auscultation revealed bronchial breath sounds in the right upper lung fields and diffuse, faint rhonchi during the expiratory phase. Her heart sounds were regular, and no murmur was noted. Hypermobility was present in the joints of the hands and arms. The remainder of the examination was normal.

Pulmonary-function testing was performed. The forced expiratory volume in 1 second $\left(\mathrm{FEV}_{1}\right)$ was $23 \%$ of the predicted value, the forced vital capacity (FVC) was $45 \%$ of the predicted value, and the ratio of $\mathrm{FEV}_{1}$ to $\mathrm{FVC}$ was $44 \%$. The total lung capacity was $108 \%$ of the predicted value, and the ratio of residual volume to total lung capacity was $65 \%$. The diffusing capacity of the lung for carbon monoxide was $26 \%$ of the predicted value.

Dr. Brent P. Little: Contrast-enhanced computed tomography (CT) of the chest revealed hyperinflation of the lungs with severe confluent emphysema and multiple apical bullae (Fig. 2A, 2B, and $2 \mathrm{C}$ ); although diffuse thickening of the bronchial wall and areas of parenchymal air trapping were present, bronchiectasis was notably absent. Normal postsurgical findings included a stump from right upper lobectomy and coil from embolization of a patent ductus arteriosus. The main pulmonary artery was enlarged, measuring $3.8 \mathrm{~cm}$ in diameter (Fig. 2D).
Dr. Mark E. Lindsay: Transthoracic echocardiography revealed dilatation of the right atrium, right ventricle, and inferior vena cava; systolic and diastolic flattening of the interventricular septum (see Video 1, available with the full text of this article at NEJM.org); and an estimated right ventricular systolic pressure of $65 \mathrm{~mm} \mathrm{Hg}$. The aortic root was not enlarged relative to the patient's body size, and the aortic valve was tricuspid. However, the aortic valve was abnormally thickened and was prolapsed, and there was moderate aortic regurgitation, which had not been present on echocardiograms obtained during infancy. Findings suggestive of constrictive pericarditis, including a septal bounce and respiratory variation across the mitral valve, were also seen.

Dr. Bartley: Empirical prednisone and amoxicillin-clavulanic acid were prescribed, and a diagnostic test was performed.

DIFFERENTIAL DIAGNOSIS

Dr. Ted M. Kremer: This patient is a 22-year-old woman with severe emphysematous obstructive lung disease, pulmonary hypertension, aortic regurgitation, and joint hypermobility. She had recurrent wheezing and findings of lobar emphysema in infancy that resulted in resection of the right upper lobe and closure of a patent ductus arteriosus by means of coil embolization when she was 2 years of age. She had an episode of pneumothorax when she was 6 years of age. Her family history is notable for joint hypermobility in her mother and seizures and developmental delays in a brother. Emphysematous obstructive lung disease is the central feature of this patient's presentation, so I will construct my differential diagnosis around this finding. However, to make a unifying diagnosis in this case, I will also need to account for several other abnormalities, including congenital heart disease, pulmonary hypertension, and joint hypermobility.

\section{EMPHYSEMATOUS OBSTRUCTIVE LUNG DISEASE}

In patients with severe emphysematous obstructive lung disease, there is a broad differential diagnosis to consider. Possible causes include postinfectious bronchiolitis obliterans, primary lung diseases, childhood interstitial lung diseases, immunodeficiency syndromes, autoimmune disorders, and connective-tissue disorders, including hypermobility syndromes. 


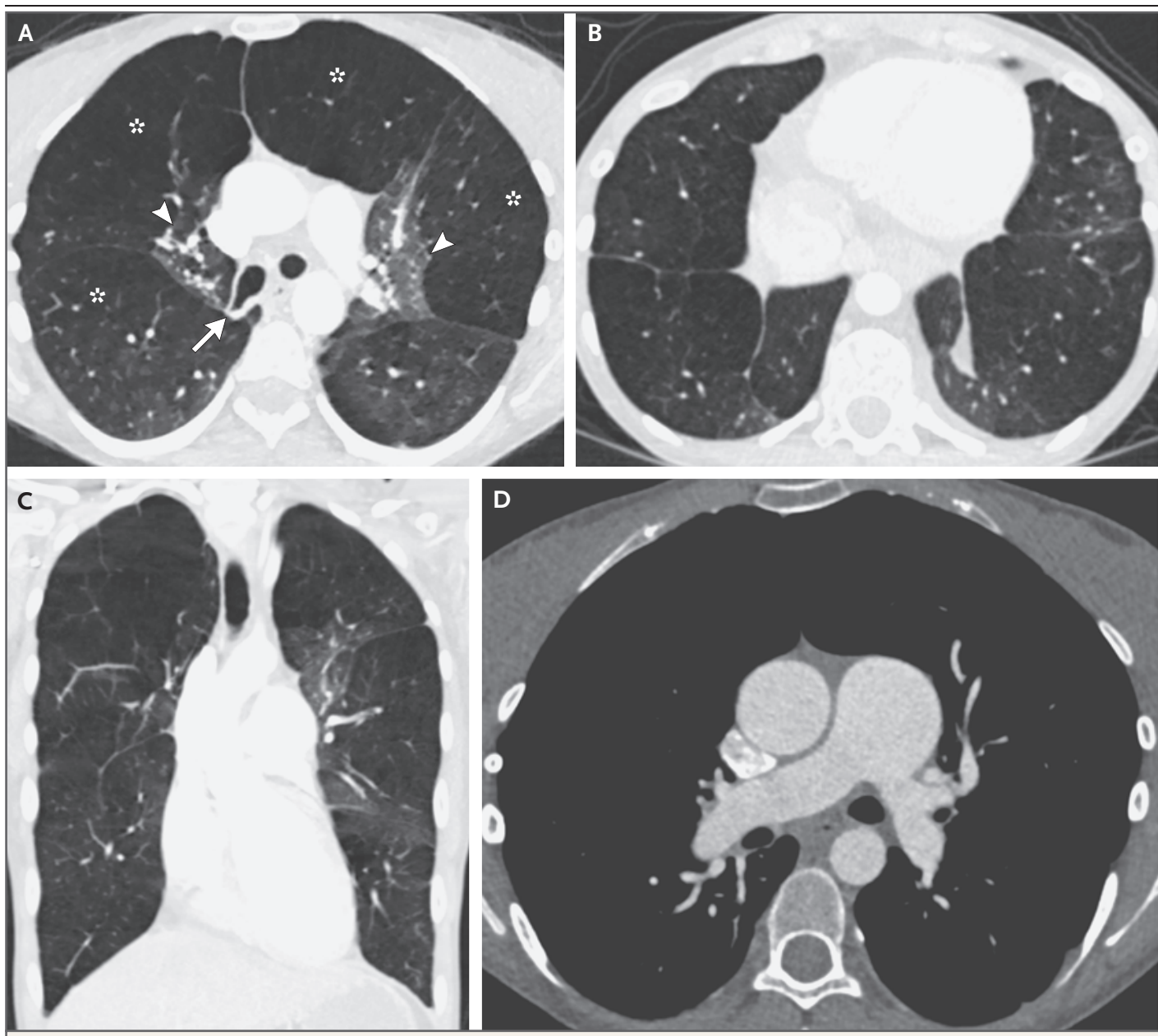

Figure 2. CT Scan of the Chest.

An axial image with lung windows at a level just below the carina (Panel A) shows diffuse emphysema with a panlobular appearance in both lungs (asterisks). A bronchial stump from right upper lobectomy performed for a previous diagnosis of congenital lobar emphysema is seen (arrow). The extensive low-attenuation regions of the lung parenchyma correspond to emphysema with air trapping, and smaller central areas of higher attenuation (arrowheads) represent less affected lung. The diameters of the pulmonary vessels in the areas of emphysema are smaller than those in the central areas, findings that represent vasoconstriction with vascular shunting toward more normal lung tissue. An axial image of the lower lungs (Panel B) shows the caudal extent of the severe emphysema. Although mild bronchial-wall thickening is present, bronchiectasis is notably absent. A corresponding coronal reformatted image (Panel C) shows the diffuse craniocaudal distribution of severe confluent emphysema. An axial image with soft-tissue windows (Panel D) shows a dilated main pulmonary artery, which measures $3.8 \mathrm{~cm}$ in diameter and is larger in diameter than the adjacent ascending aorta.

\section{POSTINFECTIOUS BRONCHIOLITIS OBLITERANS}

What can cause emphysematous lung disease associated with recurrent wheezing and bronchiolitis in infancy? Viral infection can cause postinfectious bronchiolitis obliterans with emphysematous changes similar to those of the Swyer-James syndrome. Infection with adenovirus or with other viruses, such as respiratory syncytial virus, is known to cause emphysematous syndromes. ${ }^{1}$ Although a viral infection is a plausible cause of this patient's illness and can be associated with chronic lung disease, it seems unlikely, considering the additional constellation of findings in this case, including congenital cardiovascular disease and joint hypermobility. 


\section{PRIMARY LUNG DISEASES}

Primary lung diseases associated with airflow obstruction and emphysema are strong considerations in this case. An alpha ${ }_{1}$-antitrypsin deficiency was reportedly ruled out in infancy. Although this disorder is associated with emphysematous lung disease, that manifestation usually begins in the fifth decade of life. ${ }^{2}$ This patient was born before routine newborn screening for cystic fibrosis, which also has manifestations of obstructive lung disease; however, cystic fibrosis is not typically severe in infancy, and it is unlikely to cause emphysematous lung changes at a very young age. Furthermore, other manifestations of cystic fibrosis, such as malabsorption, would probably also be present. Primary ciliary dyskinesia can progress and cause obstructive lung disease, but this pattern of events typically occurs well after infancy. None of these conditions are associated with congenital cardiac findings, but pulmonary hypertension may develop later in life as a result of chronic severe obstructive lung disease. Joint hypermobility is uncommon with these conditions.

\section{CHILDHOOD INTERSTITIAL LUNG DISEASES}

Childhood interstitial lung diseases are associated with a variety of pulmonary findings. ${ }^{3}$ Neuroendocrine-cell hyperplasia of infancy and pulmonary interstitial glycogenosis classically occur in infancy. They are associated with distinct findings on imaging, typical pathological features, and usually a more benign course than that seen in this patient. Surfactant dysfunction disorders have various outcomes. Surfactant protein B deficiency is rapidly progressive in infancy and usually leads to death. Surfactant protein C deficiency and mutation in the gene encoding ATP-binding cassette A3 ( $A B C A 3)$ may be associated with a less severe clinical course than that of surfactant protein B deficiency, but both conditions usually result in lifelong pulmonary manifestations, such as those seen in this patient. However, none of these conditions are typically associated with congenital cardiac disease, and they do not provide us with a unifying diagnosis in this case.

\section{IMMUNODEFICIENCY SYNDROMES}

Because this patient has obstructive lung disease and a history of recurring pulmonary infections, a diagnosis of an immunodeficiency syndrome is worth considering. Humoral immune responses associated with antibody deficiencies increase the risk of sinopulmonary infections and may eventually cause chronic obstructive lung disease. However, this patient has not had recurrent or chronic sinus disease. A variety of other immunodeficiency syndromes could be considered, but the severity of this patient's lung disease in infancy, together with the other findings reported in infancy in this patient, make these syndromes unlikely.

\section{AUTOIMMUNE DISORDERS}

Certain autoimmune disorders can have associated pulmonary manifestations and joint hypermobility. Inflammatory bowel disorders may be associated with bronchiectasis, bronchiolitis obliterans, and interstitial lung disease and may include joint hypermobility. ${ }^{4,5}$ These manifestations may be caused by inflammatory bowel disorders but may also be caused by the treatment of such disorders or by infections that occur in association with that treatment. In addition, celiac disease may occur with emphysema, chronic bronchitis, and hypermobility. The patient had diarrhea at the time of her recent presentation, but this symptom was not described as persistent. However, an autoimmune disorder is not likely to account for her presentation in infancy or for congenital cardiac disease.

\section{CONNECTIVE-TISSUE DISORDERS}

The findings of aortic regurgitation, pulmonary hypertension, patent ductus arteriosus, emphysema, pneumothorax, and joint hypermobility strongly suggest a connective-tissue disorder such as Marfan's syndrome, the Loeys-Dietz syndrome, or the Ehlers-Danlos syndrome. Marfan's syndrome is an autosomal dominant condition caused by mutations in the gene encoding fibrillin-1 (FBN1). ${ }^{6,7}$ At 6 years of age, after a spontaneous pneumothorax had developed, this patient underwent genetic testing; this gene was sequenced, and no mutation was identified. The Loeys-Dietz syndrome is an autosomal dominant disorder that has some phenotypic overlap with Marfan's syndrome; it is caused by disruption of genes encoding members of the canonical transforming growth factor $\beta$ (TGF- $\beta$ ) signaling cascade and has been reported to be 
associated with aortic disease, obstructive pulmonary disease, and pneumothorax. ${ }^{8}$ In contrast with Marfan's syndrome, which is monogenic, the Loeys-Dietz syndrome has been associated with multiple gene loci, including TGFBR 1 and TGFBR2, which encode the two subunits of the TGF- $\beta$ receptor (TGFBR1 and TGFBR2). The Ehlers-Danlos syndrome is associated with mutations in several different genes, with multiple genotypic and phenotypic variants, and most commonly is autosomal dominant. Type IV Ehlers-Danlos syndrome, also known as vascular Ehlers-Danlos syndrome, is associated with a defect in the gene encoding type III collagen (COL3A1). Vascular Ehlers-Danlos syndrome leads to arterial disease and may cause emphysema, pneumothorax, aortic disease, and patent ductus arteriosus. ${ }^{9-12}$ This patient's disease in young adulthood has manifestations that suggest an Ehlers-Danlos syndrome phenotype, but the onset in infancy reduces the likelihood of this possibility.

In recent years, a disorder similar to the Ehlers-Danlos syndrome that is caused by mutations in the gene encoding filamin A (FLNA) has been recognized. FLNA-related disease is an $\mathrm{X}$-linked dominant condition that has highly variable phenotypic features. The most common manifestations include a neurologic disorder called periventricular heterotopia (a neuronal migration defect), seizure, thrombocytopenia, gastrointestinal dysmotility, poor growth, skeletal dysplasia, and hypermobility. ${ }^{13}$ Different combinations of these findings may result in different types of FLNA-related disease; these differences are probably based on genetic variation. FLNArelated disease is frequently fatal in male patients.

There is emerging recognition of a respiratory phenotype of this genetic disorder that occurs in infancy and has unique features, including cardiovascular manifestations (aortic-valve disease and dilatation, patent ductus arteriosus, and pulmonary hypertension) and pulmonary manifestations (emphysema and lung cysts alternating with atelectasis and tracheobronchomalacia or stenosis). ${ }^{14-21}$ A recent review summarizes the cases reported in the literature to date. ${ }^{22}$ Thus far, at least 18 cases of FLNA-related disease with this respiratory phenotype have been identified in infancy. Most of the infants identified were girls, and respiratory symptoms developed at approximately 1 month of age. The outcomes were variable and included the use of oxygen support, lobectomy of severely emphysematous lobes, and ligation of a patent ductus arteriosus if present. Several patients underwent lung transplantation, one patient had a heart transplantation, and a small number of patients died from severe lung disease. The respiratory phenotype of FLNArelated disease is now considered to be a childhood interstitial lung disease. ${ }^{21}$ The filamin A protein is thought to be important in the maturation of alveoli in embryologic life, a process that affects lung growth and development. Findings similar to bronchopulmonary dysplasia due to alveolar simplification have been noted. ${ }^{19}$ The cause of FLNA mutation seems to vary and has been linked to deletions, missense changes, duplications, and nonsense variants, all of which may have a role in the phenotypic outcome.

This patient has a clinical picture that strongly suggests an FLNA mutation characterized by diffuse emphysematous lung disease with severe airflow obstruction that began in infancy, as well as congenital cardiac manifestations (aortic disease, patent ductus arteriosus, and pulmonary hypertension) and the connective-tissue finding of joint hypermobility. Her family history raises unique questions and is probably related to this disorder. The joint hypermobility that was diagnosed in her mother may provide evidence of an X-linked condition. At this point, it is unclear whether the brother's history of seizures and developmental delays is associated with the disorder, but these conditions may be worth exploring. It would be unusual but possible for an FLNA mutation to occur in a young adult, given the description of the respiratory phenotype in recent years.

DR. TED M. KREMER'S DIAGNOSIS

Mutation in the gene encoding filamin A (FLNA).

DIAGNOSTIC TESTING

Dr. T. Bernard Kinane: This patient initially underwent genetic screening for a connective-tissue disorder when she was 6 years of age, and no mutations were identified. However, genetic panels have expanded over time, and a genetic 
panel to test for connective-tissue disorder was obtained during the current evaluation.

The test results showed a heterozygous mutation in FLNA: c.6830_6831delTT (p.Phe2277Ter). This frameshift mutation results in premature termination of transcription near an important functional domain of the filamin A protein, an important component of the cellular cytoskeleton. ${ }^{23}$ The filamin A protein links actin, which is essential to the regulation of cell shape and migration, to the cell surface. Mutations in FLNA result in a range of clinical syndromes. ${ }^{24}$ Gainof-function mutations result in skeletal dysplasia with hearing loss (otopalatodigital syndrome types I and II and the Melnick-Needles syndrome). ${ }^{25}$ Loss-of-function mutations in FLNA were first described in patients with periventricular heterotopia, which is an anomaly in which groups of neurons fail to migrate from the periventricular region to the cerebral cortex. ${ }^{26}$ Such patients usually present with seizures during the teenage years. Less commonly, microcephaly and developmental delays are seen. Since the mutations were first described, features such as dilatation of the aorta, cardiac valvular dysplasia, flexible joints, and fragile blood vessels have been increasingly recognized.

More recently, cases of FLNA mutation with pulmonary manifestations have been identified. The first case was identified in 2011. ${ }^{20}$ Since then, 17 additional case reports of lung disease have been described in the literature, but more cases are being recognized in the clinic, particularly in lung transplantation centers. ${ }^{15-22,27}$ Of the 18 patients, 15 were female, which suggests that males are more likely to be severely affected and are less likely to survive to birth. All but 1 of these patients were born at full term. All had periventricular heterotopia. The onset of lung disease occurred during the first 3 months of life in all 18 patients. In 17 patients, CT scans showed areas with lung hyperinflation, a finding that was also seen in this case. In many of the patients, whole lobes were hyperinflated and lobar emphysema was noted. In total, 10 of the patients had a patent ductus arteriosus, and 4 had pulmonary hypertension. A total of 8 patients died or had lung transplantation. Most of these patients had mutations that occurred near important functional domains of the molecule, such as the actin-binding domain and the do- main that is important for dimerization of these molecules. In this case, the mutation occurred near an area that is important for both integrin binding and dimerization. In the case reports, 13 patients had a duplication or deletion with a resulting frameshift mutation, 1 had a nonsense mutation, and 4 had a missense mutation. There was no apparent association between mutation type and outcome, but the number of cases is small. Overall, this patient had a typical pulmonary presentation and phenotype for a patient with an FLNA mutation.

GENETIC SCREENING

Ms. Megan H. Hawley: For patients with FLNA mutations, screening recommendations would generally include a baseline neurologic evaluation, referral to an epilepsy specialist if seizures occur, and cardiovascular imaging studies, such as magnetic resonance angiography to assess the risk of stroke and echocardiography to investigate whether congenital cardiac anomalies or aortopathy are present. ${ }^{28}$ To date, there are no specific recommendations for pulmonary assessment. However, an initial pulmonary evaluation could be considered for affected relatives when pulmonary disease is present in the proband.

None of the patient's family members had clinical features diagnostic of periventricular heterotopia; however, her family history suggested that the variant may have been maternally inherited. The patient's mother reported a history of recurrent bronchitis, hypertensive crisis, edema, and mild joint hypermobility. The patient's maternal grandmother was reported to have joint pain and asthma and had several siblings who had died early in infancy. The exact cause of death and the sex of these children were unknown. Although the maternal family history was somewhat suggestive of this disorder, the patient's mother tested negative for the c.6830_6831delTT (p.Phe2277Ter) FLNA variant. Because it is unlikely that the patient's father carries the variant, given that affected males are likely to have a severe phenotype, one can conclude that the variant most likely occurred as a new mutation in the patient that was not inherited from either parent, which is not uncommon for disease-causing mutations in FLNA. ${ }^{29}$ 
Each female offspring of the patient would have a $50 \%$ chance of inheriting the mutation, although the expected clinical presentation of those offspring is difficult to predict. Because the majority of loss-of-function variants in FLNA lead to perinatal death in male offspring, pregnancies with affected male offspring would most likely result in spontaneous abortion. The possibility of live birth of a severely affected male child is low but has been reported. ${ }^{13}$

Dr. Virginia M. Pierce (Pathology): Dr. Kinane, would you tell us what happened with this patient?

Dr. Kinane: When we evaluated this patient, she had worsening pulmonary hypertension, with an estimated right ventricular systolic pressure of $65 \mathrm{~mm} \mathrm{Hg}$. She went back to her parents' home with a plan for urgent cardiology consultation. Since her evaluation at this hospital, she has been treated with oxygen and nitric oxide and subsequently has begun receiving treatment with sildenafil and bosentan. She is now also receiving supplemental oxygen at night.

\section{FINAL DIAGNOSIS}

Interstitial lung disease associated with FLNA mutation.

This case was presented at Pediatric Grand Rounds.

Dr. Lindsay reports serving as a fellow at Third Rock Ventures, for which no fees are received; Dr. Kinane, receiving fees from Sarepta Therapeutics, fees for serving on a data and safety monitoring board from Genzyme and Sanofi US Services, fees for serving as an expert witness from Weathington McGrew and W. Carl Reynolds, and grant support and fees for serving as an expert witness from ClinicalMind; and Dr. Mino-Kenudson, receiving consulting fees from Merrimack Pharmaceuticals and H3 Biomedicine. No other potential conflict of interest relevant to this article was reported.

Disclosure forms provided by the authors are available with the full text of this article at NEJM.org.

\section{REFERENCES}

1. Colom AJ, Teper AM, Vollmer WM, Diette GB. Risk factors for the development of bronchiolitis obliterans in children with bronchiolitis. Thorax 2006;61: 503-6.

2. The Alpha 1-Antitrypsin Deficiency Registry Study Group. A registry of patients with severe deficiency of alpha 1-antitrypsin: design and methods. Chest 1994;106:1223-32.

3. Kurland G, Deterding RR, Hagood JS, et al. An official American Thoracic Society clinical practice guideline: classification, evaluation, and management of childhood interstitial lung disease in infancy. Am J Respir Crit Care Med 2013; 188:376-94.

4. Black H, Mendoza M, Murin S. Thoracic manifestations of inflammatory bowel disease. Chest 2007;131:524-32.

5. Vounotrypidis P, Efremidou E, Zezos $\mathrm{P}$, et al. Prevalence of joint hypermobility and patterns of articular manifestations in patients with inflammatory bowel disease. Gastroenterol Res Pract 2009;2009: 924138.

6. Pepe G, Giusti B, Sticchi E, Abbate R, Gensini GF, Nistri S. Marfan syndrome: current perspectives. Appl Clin Genet 2016; 9:55-65.

7. Wood JR, Bellamy D, Child AH, Citron KM. Pulmonary disease in patients with Marfan syndrome. Thorax 1984;39: 780-4.

8. Attias D, Stheneur C, Roy C, et al. Comparison of clinical presentations and outcomes between patients with TGFBR2 and FBN1 mutations in Marfan syndrome and related disorders. Circulation 2009; 120:2541-9.

9. Ayres JG, Pope FM, Reidy JF, Clark TJ. Abnormalities of the lungs and thoracic cage in the Ehlers-Danlos syndrome. Tho$\operatorname{rax} 1985 ; 40: 300-5$.

10. Cupo LN, Pyeritz RE, Olson JL, McPhee SJ, Hutchins GM, McKusick VA Ehlers-Danlos syndrome with abnormal collagen fibrils, sinus of Valsalva aneurysms, myocardial infarction, panacinar emphysema and cerebral heterotopias. Am J Med 1981;71:1051-8.

11. Dowton SB, Pincott S, Demmer L. Respiratory complications of Ehlers-Danlos syndrome type IV. Clin Genet 1996;50: 510-4.

12. Ruggeri P, Calcaterra S, Girbino G. Bullous emphysema as first presentation of Ehlers-Danlos syndrome in monozygotic twins. Respir Med Case Rep 2014; 14:40-2.

13. Cannaerts E, Shukla A, Hasanhodzic $M$, et al. FLNA mutations in surviving males presenting with connective tissue findings: two new case reports and review of the literature. BMC Med Genet 2018;19:140.

14. Bickel S, Siefman M, Eid NS. Interstitial lung disease, bronchiectasis, and asthma in a patient with filamin A, alpha (FLNA) mutation. Am J Respir Crit Care Med 2015;191:A5487. abstract.

15. Burrage LC, Guillerman RP, Das S, et al. Lung transplantation for FLNA-associated progressive lung disease. J Pediatr 2017;186:118-23 e6.

16. de Wit MC, Tiddens HA, de Coo IF
Mancini GM. Lung disease in FLNA mutation: confirmatory report. Eur J Med Genet 2011;54:299-300.

17. Eltahir S, Ahmad KS, Al-Balawi MM, et al. Lung disease associated with filamin A gene mutation: a case report. J Med Case Rep 2016;10:97.

18. Case Records of the Massachusetts General Hospital (Case 4-2017). N Engl J Med 2017;376:562-74.

19. Lord A, Shapiro AJ, Saint-Martin C, Claveau M, Melançon S, Wintermark P. Filamin A mutation may be associated with diffuse lung disease mimicking bronchopulmonary dysplasia in premature newborns. Respir Care 2014;59(11): e171-e177.

20. Masurel-Paulet A, Haan E, Thompson EM, et al. Lung disease associated with periventricular nodular heterotopia and an FLNA mutation. Eur J Med Genet 2011; 54:25-8.

21. Shelmerdine SC, Semple T, Wallis C, et al. Filamin A (FLNA) mutation - a newcomer to the childhood interstitial lung disease (ChILD) classification. Pediatr Pulmonol 2017;52:1306-15.

22. Sasaki E, Byrne AT, Phelan E, Cox DW, Reardon W. A review of filamin A mutations and associated interstitial lung disease. Eur J Pediatr 2019;178:121-9.

23. Stossel TP, Condeelis J, Cooley L, et al. Filamins as integrators of cell mechanics and signalling. Nat Rev Mol Cell Biol 2001;2:138-45.

24. Reinstein E, Frentz S, Morgan T, et al. Vascular and connective tissue anomalies associated with X-linked periventricular 
heterotopia due to mutations in filamin A. Eur J Hum Genet 2013;21:494-502.

25. Robertson SP. Otopalatodigital syndrome spectrum disorders: otopalatodigital syndrome types 1 and 2, frontometaphyseal dysplasia and Melnick-Needles syndrome. Eur J Hum Genet 2007;15:3-9. 26. Parrini E, Ramazzotti A, Dobyns WB, et al. Periventricular heterotopia: phenotypic heterogeneity and correlation with filamin A mutations. Brain 2006;129: 1892-906.

27. Calcaterra V, Avanzini MA, Mantelli $\mathrm{M}$, et al. A case report on filamin A gene mutation and progressive pulmonary disease in an infant: a lung tissued derived mesenchymal stem cell study. Medicine (Baltimore) 2018;97(50):e13033.

28. Chen MH, Walsh CA. FLNA-related periventricular nodular heterotopia. In:
Adam MP, Ardinger HH, Pagon RA, et al., eds. GeneReviews. Seattle: University of Washington, 2015.

29. Lange M, Kasper B, Bohring A, et al. 47 Patients with FLNA associated periventricular nodular heterotopia. Orphanet J Rare Dis 2015;10:134.

Coppright $\odot 2019$ Massachusetts Medical Society.

JOURNAL ARCHIVE AT NEJM.ORG

Every article published by the Journal is now available at NEJM.org, beginning with the first article published in January 1812. The entire archive is fully searchable, and browsing of titles and tables of contents is easy and available to all. Individual subscribers are entitled to free 24-hour access to 50 archive articles per year. Access to content in the archive is available on a per-article basis and is also being provided through many institutional subscriptions.

The New England Journal of Medicine 